\title{
Identification of dimeric anthocyanins and new oligomeric pigments in red wine by means of HPLC-DAD-ESI/MS ${ }^{n}$
}

\author{
Cristina Alcalde-Eon, María Teresa Escribano-Bailón, Celestino Santos-Buelga and \\ Julián C. Rivas-Gonzalo*
}

Grupo de Investigación en Polifenoles, Departamento de Química Analítica, Nutrición y Bromatología, Facultad de Farmacia. Universidad de Salamanca, Campus Miguel de Unamuno, 37007 Salamanca, Spain

Received 14 November 2006; Accepted 21 February 2007

\begin{abstract}
High-pressure liquid chromatography-diode array detector-electrospray ionisation/ion trap mass spectrometry (HPLC-DAD-ESI/MS ${ }^{n}$ ) analyses carried out in red wine fractions revealed the existence of dimeric anthocyanins $\left(\mathrm{A}-\mathrm{A}^{+}\right)$, previously detected in grape skin, and allowed the detection and identification, for the first time, of other derived oligomers. The structure of these compounds was characterised according to their MS $^{n}(n=1-4)$ fragmentation patterns. The newly detected oligomers consisted of a flavanol, (epi)catechin or (epi)gallocatechin, linked through its $\mathrm{C}_{4}$ position to the nucleophilic positions of the upper unit of a dimeric anthocyanin $\left(\mathrm{F}-\mathrm{A}-\mathrm{A}^{+}\right)$. All the compounds contained malvidin as one of the anthocyanin subunits, whereas the other anthocyanin moiety could be either delphinidin, cyanidin, petunidin, peonidin or malvidin. With the fractionation method employed, the dimeric anthocyanins eluted in the same fractions as the monomeric anthocyanins. However, the new $\mathrm{F}-\mathrm{A}-\mathrm{A}^{+}$oligomers were found in the same fractions as $\mathrm{F}-\mathrm{A}^{+}$dimers, which might indicate a structural similarity between both types of compounds. ESI/MS ${ }^{n}$ analyses, coupled or not to HPLC, showed to be a useful and powerful tool for detecting and identifying these oligomers in wines, which usually elute from reversed-phase columns as humps and/or are overlapped by the peaks of other compounds. The detection of these oligomeric pigments in wine has provided more information about the complex pigments responsible for the elevation of the base line observed in the chromatograms of wines and has also revealed that oligomeric anthocyanins can take part in the reactions usually undergone by monomeric anthocyanins. Copyright $\odot 2007$ John Wiley \& Sons, Ltd.
\end{abstract}

KEYWORDS: anthocyanins; oligomeric pigments; electrospray mass spectrometry; red wine; flavanol - anthocyanin condensation products

\section{INTRODUCTION}

The colour of red wine changes, during its ageing, from the purple-red hues typical of young wines to the red-brick ones characteristic of older wines. The anthocyanins extracted from red grapes are responsible for the colour in the first stages of the wine's life. During wine maturation and ageing, an important decrease in their levels occurs and, as reported earlier by Somers, ${ }^{1}$ they are progressively replaced by more stable polymeric pigments. The first studies on grape anthocyanins were carried out by one- and two-dimensional paper chromatography, which allowed the detection of up to 21 different compounds. ${ }^{2}$ The use of high-pressure liquid chromatography (HPLC) with reversed-phase columns provided a faster and improved separation of these compounds and

*Correspondence to: Julián C. Rivas-Gonzalo, Grupo de Investigación en Polifenoles, Departamento de Química Analítica Nutrición y Bromatología, Facultad de Farmacia. Universidad de Salamanca, Campus Miguel de Unamuno, 37007 Salamanca, Spain E-mail: jcrivas@usal.es allowed their tentative identification based on their retention characteristics. ${ }^{3,4}$ Further, coupling to diode array photometric detectors permitted the simultaneous recording of chromatograms at different wavelengths as well as the acquisition of the UV-vis spectra of the peaks without requiring previous isolation of the compounds, thus increasing the possibilities of identification. ${ }^{5-7}$ Fast atom bombardment mass spectrometry (FABMS) has also been successfully employed for the identification of the isolated anthocyanins. ${ }^{8}$ However, it was replaced by softer ionisation techniques such as atmospheric pressure ionisation (API) techniques (electrospray ionisation, ESI, and atmospheric pressure chemical ionisation, APCI, above all). These techniques permit the use of highly polar solvents under acidic $\mathrm{pH}$ conditions and operate at low temperatures, avoiding anthocyanin degradation and keeping the analytes unfragmented, ${ }^{9-11}$ thus producing molecular ions in high abundance. API techniques and especially ESI/MS have been largely employed over the last decade as interfaces coupled to HPLC. In addition, collision induced dissociation (CID) tandem mass (MS/MS) spectrometry and 
$\mathrm{MS}^{n}$ analyses, which allow the sequential fragmentation of a given molecular ion, provide information for the identification of anthocyanins based on their fragmentation patterns. ${ }^{12}$ This information is very useful to differentiate compounds with similar chromatographic and UV-vis spectral features and possess the same $m / z$ ratios. Thus, data provided by high performance liquid chromatography-diode array detectorelectrospray ionisation/ion trap mass spectrometry (HPLCDAD-ESI $/ \mathrm{MS}^{n}$ ) analyses (chromatographic behaviour, UVvis spectra, molecular ion and fragmentation pattern) yielded decisive information to identify anthocyanins present in complex mixtures like wines, as well as to characterise new anthocyanin-derivatives formed during wine making and ageing. Using these techniques, several authors have been able to detect in wines of different varieties a large number of pigments belonging to different families. ${ }^{13-20}$ In spite of the numerous compounds nowadays identified, there is still lack of information about the oligomeric and polymeric pigments. In the pyranoanthocyanin family, oligomeric compounds originated from the reaction between malvidin-based anthocyanins and different vinylprocyanidin dimers have been detected not only in wine-like model solutions ${ }^{21}$ but also in wines. ${ }^{18,22}$ Compounds very much related to these pyranoanthocyanins but even more complex have been isolated from Port red wine and identified as compounds originating from the reaction between anthocyanin-pyruvic acid adducts and vinylflavanols. ${ }^{23}$ Also, some oligomers resulting from acetaldehyde-mediated and direct flavanol-anthocyanin condensation reactions have been described. ${ }^{24,25}$ The formation in model solutions of oligomers possessing one or two anthocyanins ethyl-linked with one to three flavanol units was shown by Es-Safi and co-workers. ${ }^{24}$ Hayasaka and Kennedy ${ }^{25}$ demonstrated by direct infusion ES-MS and ES-MS/MS, and confirmed by LC-MS, the presence in wine of A-T and T- $\mathrm{A}^{+}$direct condensation products consisting in one anthocyanin (A) combined with one or more proanthocyanidins $(\mathrm{T})$. They were able to detect polymers up to octamers (one anthocyanin and seven proanthocyanidins). Recently, the presence of directly linked anthocyanin dimers and trimers has been demonstrated in grape skins. ${ }^{26}$

Despite all of this progress, more studies are still required in order to draw conclusions about the precise nature of the complex pigment fraction in red wines that elutes as a 'hump' at the end of the HPLC chromatograms, observed not only in non-fractionated wines but also in the fractions obtained from them. It is assumed that this elevation of the base line is due to the oligomeric and polymeric pigmented material, which, because of their complex nature, do not elute as defined peaks in the chromatographic conditions usually employed.

In previous work of our group a method was optimised and successfully applied for the fractionation of the pigment material of red wines. ${ }^{17,27}$ The purpose of the present study was to investigate the chemical nature of oligomeric compounds present in fractions mainly consisting of anthocyanins and flavanol-anthocyanin condensation products. With this aim, HPLC-DAD-ESI/MS and ESI/MS ${ }^{n}$ analyses of the fractions have been carried out and complemented in some cases with the data obtained after the direct infusion of the fractions in the ESI source.

\section{MATERIALS AND METHODS}

\section{Samples}

Wines of four consecutive vintages from Vitis vinifera L. $c v$ Tempranillo grapes from the same vineyard and elaborated by Bodegas RODA S.A. (DO Rioja, Haro, Spain) were fractionated and analysed at different stages of making and ageing. Alcoholic and malolactic fermentation took place spontaneously, and after the latter, the wines were transferred into new French oak barrels (medium toast) and maintained in them for approximately 14 months. Then, they were bottled and stored in the cellar at $14^{\circ} \mathrm{C}$. Samples collected during maturation and ageing in the barrel and during ageing in the bottles were considered in this study.

Samples were analysed by HPLC-DAD-ESI/MS ${ }^{n}$ before their fractionation. For this purpose $1 \mathrm{ml}$ of wine was diluted to a final volume of $5 \mathrm{ml}$ with acidified water $(\mathrm{HCl}, \mathrm{pH}=0.5)$ and filtered through a $0.45-\mu \mathrm{m}$ Millex ${ }^{\circledR}$ syringe-driven filter unit (Millipore Corporation, Bedford, MA, USA) prior to their injection in to the HPLC system.

\section{Sample fractionation}

Fifteen milliliters of each wine sample was fractionated with the method previously developed and employed by Alcalde-Eon and co-workers. ${ }^{17}$ The different eluates were not gathered in two fractions but were studied like independent fractions, in order to achieve better identification of the compounds. All these fractions underwent the same treatment as already reported ${ }^{17}$ and were then analysed by HPLC-DAD-ESI/MS ${ }^{n}$ techniques.

\section{HPLC-DAD-ESI/MS ${ }^{n}$}

Analyses were performed in a Hewlett-Packard 1100 series liquid chromatograph. An AQUA C18 reversed-phase, $5 \mu \mathrm{m}$, $150 \mathrm{~mm} \times 4.6 \mathrm{~mm}$ column (Phenomenex ${ }^{\circledR}$, Torrance, CA, USA) thermostatted at $35^{\circ} \mathrm{C}$ was used. The HPLC-DAD conditions have been previously employed with satisfactory results in our laboratory in the analysis of wine samples. ${ }^{17-19,27}$ The solvents were: $0.1 \%$ trifluoroacetic acid in water (A) and 100\% HPLC-grade acetonitrile (B), establishing the following gradient: isocratic $10 \% \mathrm{~B}$ for $5 \mathrm{~min}$, from 10 to $15 \%$ B for $15 \mathrm{~min}$, isocratic $15 \%$ B for $5 \mathrm{~min}$, from 15 to $18 \%$ B for $5 \mathrm{~min}$, and from 18 to $35 \%$ B for $20 \mathrm{~min}$, at a flow rate of $0.5 \mathrm{ml} / \mathrm{min}$. Detection was carried out at $520 \mathrm{~nm}$ as the preferred wavelength. Spectra were recorded from 220 to $600 \mathrm{~nm}$.

The mass analyses were performed using a Finnigan ${ }^{\mathrm{TM}}$ LCQ ion trap instrument (Thermoquest, San Jose, CA, USA) equipped with an API source using an electrospray ionisation (ESI) interface. The LC system was connected to the probe of the mass spectrometer via the UV cell outlet. Nitrogen was used as sheath and auxiliary gas. The sheath gas flow was $1.21 / \mathrm{min}$ and the auxiliary gas flow, $61 / \mathrm{min}$. The capillary voltage was $4 \mathrm{~V}$ and the capillary temperature $195^{\circ} \mathrm{C}$. The source voltage was $4.5 \mathrm{kV}$. Spectra were recorded in positive ion mode between $\mathrm{m} / \mathrm{z} 120$ and 1500 . The mass spectrometer 
was programmed to do a series of three consecutive scans: a full mass, an $\mathrm{MS}^{2}$ scan of the most abundant ion in the full mass and an $\mathrm{MS}^{3}$ of the most abundant ion in the $\mathrm{MS}^{2}$. The normalised energy of collision was $45 \%$.

\section{Direct infusion analysis}

In order to confirm the fragmentation pattern observed for some compounds in the HPLC-DAD-ESI/MS ${ }^{n}$ analyses and to know those of other compounds that were not possible to obtain in those analyses, some fractions were also analysed by direct infusion in the mass spectrometer. Detection of the selected molecular ions in the fractions was first made using a selected ion monitoring (SIM) mode analysis, and then they were successively fragmented. The major fragment ions (and in some cases, other interesting ones) in the $\mathrm{MS}^{2}$ and $\mathrm{MS}^{3}$ spectra were selected and fragmented in the $\mathrm{MS}^{3}$ and $\mathrm{MS}^{4}$ analyses, respectively. The normalised energy of collision employed in most cases was $45 \%$, but in some analyses it was adjusted to higher or lower values in order to produce more or less fragmentation. The isolation width was set at $2.0 \mathrm{u}$. The gas flows, capillary voltage and temperature and the source voltage were the same as mentioned above for the HPLC-DAD-ESI/MS ${ }^{n}$ analyses.

\section{Materials}

All solvents were HPLC quality, and ultrapure water was produced by a Direct- $\mathrm{Q}^{\mathrm{TM}} 3$ system (Millipore Corporation, Bedford, MA, USA). Sodium bisulfite (Acros Organics, New Jersey, USA) was of analysis quality.

\section{RESULTS AND DISCUSSION}

In the wine fractions obtained corresponding to anthocyanins and flavanol-anthocyanin direct condensation pigments, compounds showing the same $m / z$ as some of the oligomeric anthocyanins described in grape skins by Vidal and coworkers ${ }^{26}$ were detected, as well as other compounds with higher $m / z$ ratios ranging from 1229 to 1289 .

\section{Dimeric anthocyanins: $\mathrm{A}-\mathrm{A}^{+}$}

Using direct ESI/MS infusion, Vidal and co-workers ${ }^{26}$ were able to detect, in grape skin, dimeric and trimeric anthocyanins, either acylated or not with one molecule of $p$-coumaric acid. Four of the non-acylated anthocyanin dimers were also detected by Salas and co-workers ${ }^{28}$ in a wine fraction containing almost only polymeric pigments, obtained after fractionation of a wine by high-speed countercurrent chromatography.

In the present study, molecular ions showing $\mathrm{m} / \mathrm{z}$ corresponding to dimers containing two anthocyanin glucosides or one glucoside and one $p$-coumaroylglucoside were detected. To be precise, in the fractions mostly constituted by anthocyanins, signals at $m / z$ 957, 941, 971, 955, 985, 1103, $1087,1117,1101$ and 1131 were observed. These $m / z$ ratios were consistent with dimers containing one molecule of malvidin $(\mathrm{Mv})$, one of delphinidin (Dp) and two glucoses $(\mathrm{m} / \mathrm{z}$ 957); one malvidin, one cyanidin (Cy) and two glucoses $(\mathrm{m} / \mathrm{z}$ 941); one malvidin, one petunidin $(\mathrm{Pt})$ and two glucoses $(m / z$ 971); one malvidin, one peonidin (Pn) and two glucoses $(m / z$ 955); two malvidins and two glucoses $(m / z$ 985) and the corresponding compounds showing one additional $p$-coumaroyl residue $(m / z$ at $1103,1087,1117,1101$ and 1131$)$.

All these compounds were detected as singly charged ions, which implied that only one of the two anthocyanin units was in its flavylium form. Studies on anthocyanin-tannin direct condensation products have revealed that the anthocyanin is in its flavylium form when it is located in the lowest position or base unit ( $\mathrm{T}-\mathrm{A}^{+}$-type dimers), whereas when it is in the upper or top unit (A-T-type dimers), it is either in flavene or flavan form depending on the type of linkage (B- or A-type, respectively) between the two flavonoid moieties. ${ }^{25,29-31}$ On the basis of these observations, it could be expected that in the oligomeric anthocyanins, the flavylium form also constitutes the lowest or base unit, while the upper anthocyanin unit would be either of a flavan (A-type linkage) or a flavene form (B-type linkage). The type of linkage between anthocyanin units seems difficult to be established taking into account only the mass spectral data; an NMR analysis of these anthocyanin oligomers would be needed to achieve it.

The fragmentation patterns of these compounds obtained in the HPLC-DAD-ESI/MS ${ }^{n}$ and direct infusion ESI/MS ${ }^{n}$ analyses were helpful in confirming the proposed identity. Direct infusion ESI/MS ${ }^{n}$ (up to $\mathrm{MS}^{4}$ ) analyses were employed in this study in order to determine and confirm the fragmentation pattern of the compounds, especially of

Table 1. Retention times, molecular and fragment ions of the dimeric anthocyanins detected in the samples analysed. The fragment ions are listed in the order of abundance, i.e. the first one is the most abundant fragment obtained in the respective $\mathrm{MS}^{n}$ analysis and is the one that is fragmented in the subsequent analysis

\begin{tabular}{|c|c|c|c|c|c|}
\hline$\left(\mathbf{M}^{+}\right) m / z$ & $\begin{array}{c}t_{\mathrm{R}} \\
(\mathrm{min})\end{array}$ & $\begin{array}{c}\mathrm{MS}^{2} \\
\text { fragment ions } \\
m / z\end{array}$ & $\begin{array}{c}\mathrm{MS}^{3} \\
\text { fragment ions } \\
m / z\end{array}$ & $\begin{array}{c}\mathrm{MS}^{4} \\
\text { fragment ions } \\
m / z\end{array}$ & $\begin{array}{l}\text { Proposed } \\
\text { identity }\end{array}$ \\
\hline 957 & $27-37$ & $\begin{array}{l}795\left[\mathrm{M}^{+}-162\right] \\
633\left[\mathrm{M}^{+}-324\right]\end{array}$ & $\begin{array}{l}633\left[\mathrm{MS}^{+}-162\right] \\
615\left[\mathrm{MS}^{+}-180\right] \\
507\left[\mathrm{MS}^{+}-288\right] \\
331\left[\mathrm{MS}^{+}-464\right] \\
467\left[\mathrm{MS}^{+}-328\right] \\
303\left[\mathrm{MS}^{+}-492\right] \\
439\left[\mathrm{MS}^{+}-356\right]\end{array}$ & $\begin{array}{c}507\left[\mathrm{MS3}^{+}-126\right] \\
303\left[\mathrm{MS3}^{+}-330\right] \\
331\left[\mathrm{MS3}^{+}-302\right] \\
615\left[\mathrm{MS}^{+}-18\right]\end{array}$ & Dimer MvDp $2 C$ \\
\hline
\end{tabular}


Table 1. (Continued)

\begin{tabular}{|c|c|c|c|c|c|}
\hline$\left(\mathrm{M}^{+}\right) m / z$ & $\begin{array}{c}t_{\mathrm{R}} \\
(\mathrm{min})\end{array}$ & $\begin{array}{c}\mathrm{MS}^{2} \\
\text { fragment ions } \\
m / z\end{array}$ & $\begin{array}{c}\mathrm{MS}^{3} \\
\text { fragment ions } \\
m / z\end{array}$ & $\begin{array}{c}\mathrm{MS}^{4} \\
\text { fragment ions } \\
m / z\end{array}$ & $\begin{array}{c}\text { Proposed } \\
\text { identity }\end{array}$ \\
\hline 941 & $32-41$ & $\begin{array}{l}779\left[\mathrm{M}^{+}-162\right] \\
617\left[\mathrm{M}^{+}-324\right]\end{array}$ & $\begin{array}{l}617\left[\mathrm{MS}^{+}-162\right] \\
599\left[\mathrm{MS}^{+}-180\right] \\
449\left[\mathrm{MS}^{+}-330\right]\end{array}$ & $\begin{array}{c}599\left[\mathrm{MS}^{+}-18\right] \\
491\left[\mathrm{MS}^{+}-126\right] \\
331\left[\mathrm{MS}^{+}-286\right]\end{array}$ & Dimer MvCy 2G \\
\hline 971 & $33-42$ & $\begin{array}{l}809\left[\mathrm{M}^{+}-162\right] \\
647\left[\mathrm{M}^{+}-324\right] \\
331\left[\mathrm{M}^{+}-640\right]\end{array}$ & $\begin{array}{l}647\left[\mathrm{MS}^{+}-162\right] \\
629\left[\mathrm{MS}^{+}-180\right] \\
331\left[\mathrm{MS}^{+}-478\right] \\
317\left[\mathrm{MS}^{+}-492\right] \\
302\left[\mathrm{MS}^{+}-507\right] \\
521\left[\mathrm{MS}^{+}-288\right]\end{array}$ & $\begin{array}{c}629\left[\mathrm{MS}^{+}-18\right] \\
521\left[\mathrm{MS}^{+}-126\right] \\
317\left[\mathrm{MS3}^{+}-330\right] \\
331\left[\mathrm{MS3}^{+}-316\right]\end{array}$ & Dimer MvPt 2G \\
\hline 955 & $37-42$ & $\begin{array}{l}793\left[\mathrm{M}^{+}-162\right] \\
631\left[\mathrm{M}^{+}-324\right]\end{array}$ & $\begin{array}{l}631\left[\mathrm{MS}^{+}-162\right] \\
613\left[\mathrm{MS}^{+}-180\right] \\
505\left[\mathrm{MS}^{+}-288\right] \\
331\left[\mathrm{MS}^{+}-462\right] \\
301\left[\mathrm{MS}^{+}-492\right] \\
437\left[\mathrm{MS}^{+}-356\right] \\
667\left[\mathrm{MS}^{+}-126\right]\end{array}$ & $\begin{array}{c}613\left[\mathrm{MS3}^{+}-18\right] \\
505\left[\mathrm{MS}^{+}-126\right] \\
301\left[\mathrm{MS}^{+}-330\right] \\
331\left[\mathrm{MS}^{+}-300\right]\end{array}$ & Dimer MvPn 2G \\
\hline 985 & $38-43$ & $\begin{array}{l}823\left[\mathrm{M}^{+}-162\right] \\
661\left[\mathrm{M}^{+}-324\right]\end{array}$ & $\begin{array}{l}661\left[\mathrm{MS}^{+}-162\right] \\
643\left[\mathrm{MS}^{+}-180\right] \\
331\left[\mathrm{MS}^{+}-492\right] \\
535\left[\mathrm{MS}^{+}-288\right] \\
467\left[\mathrm{MS}^{+}-356\right]\end{array}$ & $\begin{array}{c}535\left[\mathrm{MS3}^{+}-126\right] \\
643\left[\mathrm{MS3}^{+}-18\right] \\
331\left[\mathrm{MS3}^{+}-330\right]\end{array}$ & Dimer MvMv 2G \\
\hline 1103 & 43 & $\begin{array}{l}795\left[\mathrm{M}^{+}-308\right] \\
941\left[\mathrm{M}^{+}-162\right] \\
633\left[\mathrm{M}^{+}-470\right] \\
493\left[\mathrm{M}^{+}-610\right] \\
331\left[\mathrm{M}^{+}-772\right]\end{array}$ & $\begin{array}{l}633\left[\mathrm{MS}^{+}-162\right] \\
615\left[\mathrm{MS}^{+}-180\right] \\
331\left[\mathrm{MS}^{+}-464\right] \\
303\left[\mathrm{MS}^{+}-492\right]\end{array}$ & $\begin{array}{c}615\left[\mathrm{MS3}^{+}-18\right] \\
331\left[\mathrm{MS3}^{+}-302\right] \\
507\left[\mathrm{MS3}^{+}-126\right]\end{array}$ & Dimer MvDp G-CG \\
\hline 1087 & 44 & $\begin{array}{l}779\left[\mathrm{M}^{+}-308\right] \\
925\left[\mathrm{M}^{+}-162\right] \\
617\left[\mathrm{M}^{+}-470\right]\end{array}$ & $\begin{array}{l}617\left[\mathrm{MS}^{+}-162\right] \\
599\left[\mathrm{MS}^{+}-180\right] \\
493\left[\mathrm{MS}^{+}-286\right] \\
331\left[\mathrm{MS}^{+}-448\right] \\
287\left[\mathrm{MS}^{+}-492\right]\end{array}$ & $\begin{array}{c}599\left[\mathrm{MS3}^{+}-18\right] \\
491\left[\mathrm{MS}^{+}-126\right] \\
287\left[\mathrm{MS}^{+}-330\right] \\
331\left[\mathrm{MS}^{+}-286\right]\end{array}$ & Dimer MvCy G-CG \\
\hline 1117 & 44.2 & $\begin{array}{l}809\left[\mathrm{M}^{+}-308\right] \\
955\left[\mathrm{M}^{+}-162\right] \\
647\left[\mathrm{M}^{+}-470\right] \\
493\left[\mathrm{M}^{+}-624\right]\end{array}$ & $\begin{array}{l}647\left[\mathrm{MS}^{+}-162\right] \\
629\left[\mathrm{MS}^{+}-180\right] \\
521\left[\mathrm{MS}^{+}-288\right] \\
331\left[\mathrm{MS}^{+}-478\right] \\
467\left[\mathrm{MS}^{+}-342\right]\end{array}$ & $\begin{array}{c}629\left[\mathrm{MS}^{+}-18\right] \\
317\left[\mathrm{MS}^{+}-330\right] \\
521\left[\mathrm{MS}^{+}-126\right] \\
331\left[\mathrm{MS3}^{+}-316\right]\end{array}$ & Dimer MvPt G-CG \\
\hline 1101 & 45 & $\begin{array}{l}793\left[\mathrm{M}^{+}-308\right] \\
939\left[\mathrm{M}^{+}-162\right] \\
631\left[\mathrm{M}^{+}-470\right]\end{array}$ & $\begin{array}{l}631\left[\mathrm{MS}^{+}-162\right] \\
505\left[\mathrm{MS}^{+}-288\right] \\
613\left[\mathrm{MS}^{+}-180\right] \\
467\left[\mathrm{MS}^{+}-326\right] \\
331\left[\mathrm{MS}^{+}-462\right] \\
301\left[\mathrm{MS}^{+}-492\right]\end{array}$ & $\begin{array}{c}613\left[\mathrm{MS}^{+}-18\right] \\
301\left[\mathrm{MS}^{+}{ }^{+}-330\right] \\
331\left[\mathrm{MS3}^{+}-300\right] \\
505\left[\mathrm{MS}^{+}{ }^{+}-126\right]\end{array}$ & Dimer MvPn G-CG \\
\hline 1131 & 45.2 & $\begin{array}{l}823\left[\mathrm{M}^{+}-308\right] \\
969\left[\mathrm{M}^{+}-162\right] \\
661\left[\mathrm{M}^{+}-470\right]\end{array}$ & $\begin{array}{l}661\left[\mathrm{MS}^{+}-162\right] \\
643\left[\mathrm{MS}^{+}-180\right] \\
467\left[\mathrm{MS}^{+}-356\right] \\
331\left[\mathrm{MS}^{+}-492\right]\end{array}$ & $\begin{array}{l}535\left[\mathrm{MS3}^{+}-126\right] \\
331\left[\mathrm{MS}^{+}-330\right]\end{array}$ & Dimer MvMv G-CG \\
\hline
\end{tabular}

$\mathrm{M}^{+}$, molecular ion; $\mathrm{t}_{\mathrm{R}}$, retention time for the compounds eluting as peaks, and range of time for the peaks eluting as a hump; MS2 ${ }^{+}$, major fragment ion obtained in the $\mathrm{MS}^{2}$ analysis; $\mathrm{MS}^{+}$, major fragment ion obtained in the $\mathrm{MS}^{3}$ analysis; Dp, delphinidin; Cy, cyanidin; Pt, petunidin; Pn, peonidin; Mv, malvidin; G, glucose; CG, $p$-coumaroylglucose.

those whose fragmentation could not be obtained in the HPLC-DAD-ESI/MS analysis of the fractions owing to their low levels. Some characteristic fragmentations have been observed (Table 1 and Fig. 1). In the case of the dimeric anthocyanins with two glucoses, the most abundant $\mathrm{MS}^{2}$ fragment ion originated from the loss of one glucose $(-162 \mathrm{amu})$ and a secondary one was produced after the loss of $324 \mathrm{amu}$, corresponding to the dimeric aglycone. 


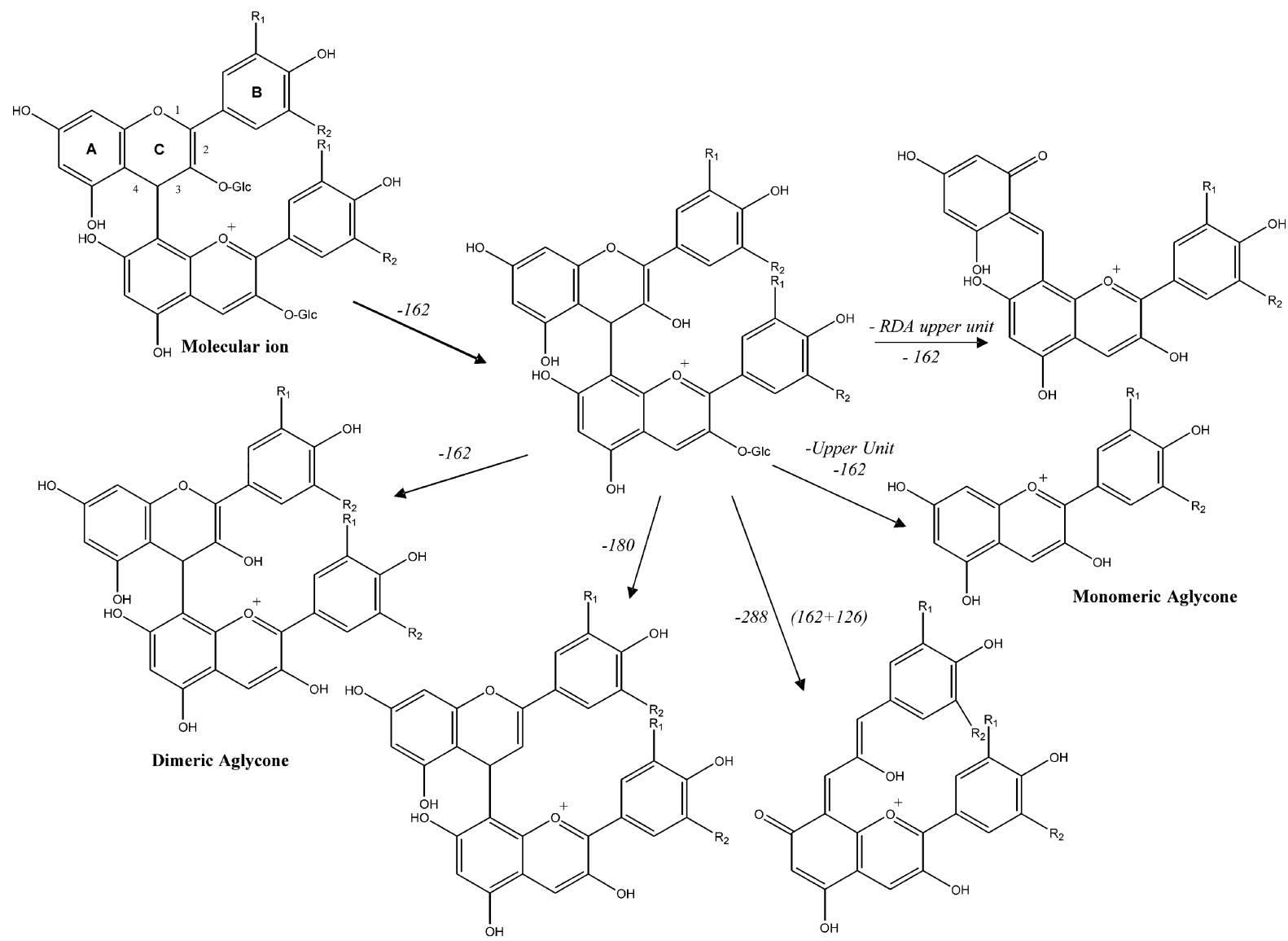

Figure 1. Fragmentation pattern of the dimeric anthocyanins. The first step is the fragmentation of the molecular ion in the $\mathrm{MS}^{2}$ analysis, yielding the monoglucosylated compound as the main fragment ion. The figure also shows the main fragment ions originating in the $\mathrm{MS}^{3}$ analysis of the former: $\mathrm{R}_{1}$ and $\mathrm{R}_{2}=\mathrm{H}, \mathrm{OH}$ or $\mathrm{OCH}_{3}$, depending on the anthocyanins involved. In this figure a B-type dimer is represented, but an A-type linkage between both anthocyanins might be also possible.

Other fragment ions were usually present in very low levels (relative abundance lower than $5 \%$, e.g. Fig. $2-\mathrm{MS}^{2}$ spectrum of the molecular ion at $m / z$ 955). In the $\mathrm{MS}^{3}$ analyses, the main $\mathrm{MS}^{2}$ fragment ion yielded the dimeric aglycone as the major ion. Other fragment ions in the $\mathrm{MS}^{3}$ spectra of almost all the compounds were those originating from the losses of 180 and 288 amu (Table 1 and Figs 1 and 2 ). The first one is attributed to the simultaneous loss of the glucose residue and a water molecule $(-162-18 \mathrm{amu})$ and the second one to the loss of the glucose residue and a phloroglucinol molecule $(-126 \mathrm{amu})$ released from the heterocyclic ring fission (HRF) in the upper or top unit (Fig. 1). This latter is one of the most common fragmentation pathways observed for B- and A-type proanthocyanidins ${ }^{32-34}$ and has also been observed in T-A $\mathrm{A}^{+31,35}$ and A-type A-T dimers. ${ }^{29,30}$ Vidal and co-workers ${ }^{26}$ also reported this in the fragmentation of the oligomeric anthocyanins detected in grape skin. HRF was also observed in the $\mathrm{MS}^{4}$ analysis, thus confirming that the loss of 288 amu does really correspond to one glucose and one phloroglucinol and not to the release of a $\mathrm{C}_{4}$-linked (epi)catechin. In the $\mathrm{MS}^{3}$ spectra, signals at $\mathrm{m} / \mathrm{z}$ corresponding to monomeric anthocyanin aglycones $(m / z$ at 303, 287, 317, 301 and 331 for Dp, Cy, Pt, Pn and $\mathrm{Mv}$, respectively) were also detected. In most cases, the ions of two anthocyanidins were observed (Table 1 and, e.g. refer in Fig. 2 the $\mathrm{MS}^{3}$ and $\mathrm{MS}^{4}$ spectra of the dimer containing Mv and Pn and two glucoses, $m / z$ 955). Taking into account that only one of the anthocyanins in the dimers is in the flavylium form and, therefore, only this one can be detected as an ion after fragmentation, the observation of the ions of both anthocyanidins suggested that dimers composed by the same anthocyanins in different configurations were simultaneously detected (i.e. in the former example, $\mathrm{Pn}-\mathrm{Mv}^{+}$and $\mathrm{Mv}-\mathrm{Pn}^{+}$), as also speculated by Vidal and co-workers ${ }^{26}$ on the basis of the observation of the RDA fission in both anthocyanins. In the direct infusion analysis, it is possible to produce at the same time the fragmentation of different molecular ions with the same $\mathrm{m} / \mathrm{z}$ ratios, since there is no previous chromatographic separation. However, we have detected simultaneously dimers with distinct relative positions of the same anthocyanins not only in the fragmentations made by direct infusion ESI-MS ${ }^{n}$ but also in those carried out after HPLC separation. This would mean that both dimers co-elute.

Under the conditions employed in this study, the RDA fission of the top unit was observed only for some of the compounds, and in most cases this loss resulted at the same time as that of a glucose moiety $(-328$ amu for Dp, -342 for $\mathrm{Pt},-326$ for Pn and -356 for Mv). It has to be noted that in dimers containing an A-type linkage, the same loss could be observed, although in such cases, the fragmentation that originated it is not strictly a retro Diels-Alder (RDA) fission. 

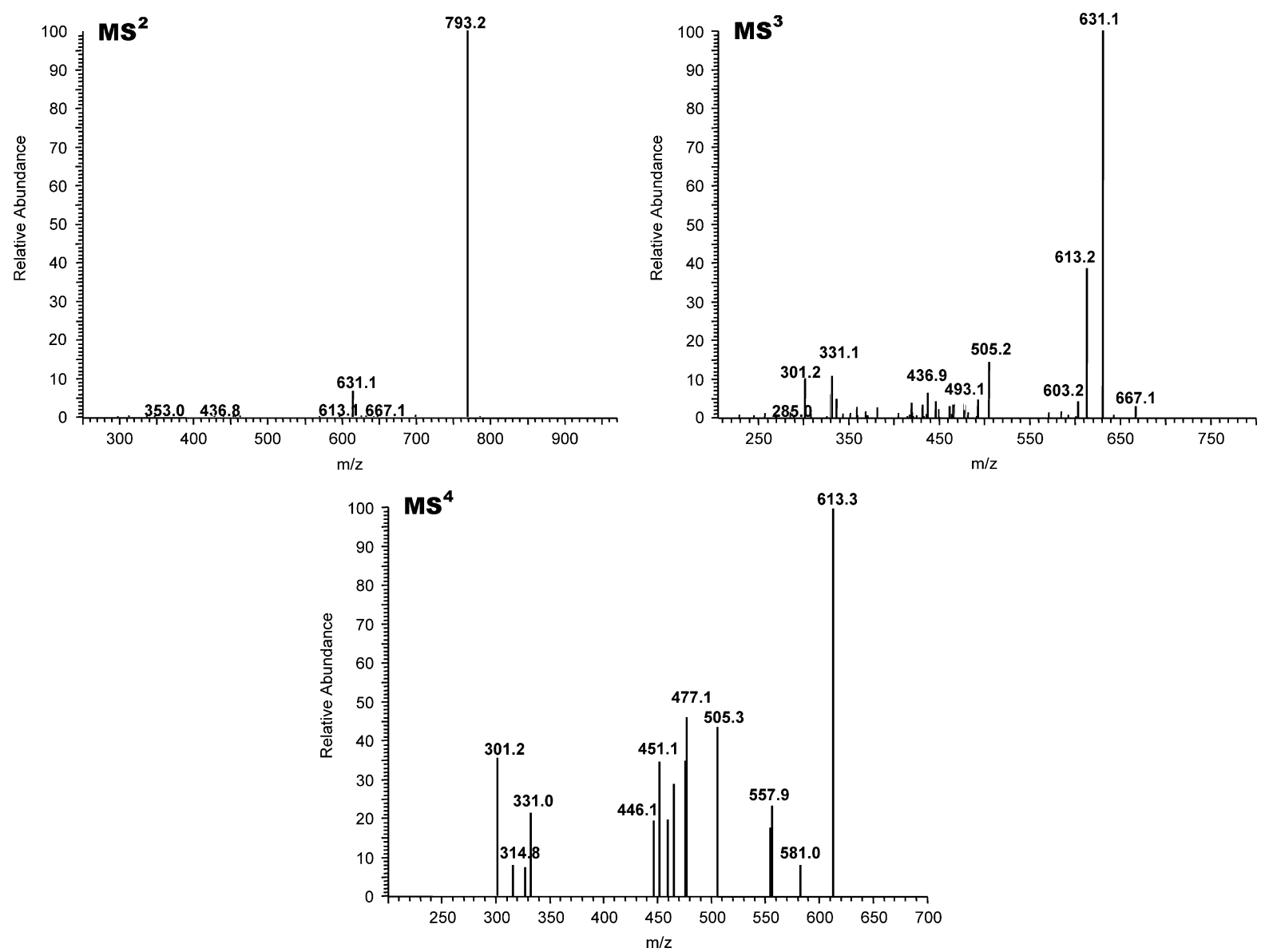

Figure 2. $\mathrm{MS}^{2}, \mathrm{MS}^{3}$ and $\mathrm{MS}^{4}$ spectra of the molecular ion at $\mathrm{m} / \mathrm{z}$ 955, composed of peonidin, malvidin and two glucoses. $\mathrm{MS}^{2}$ and $\mathrm{MS}^{3}$ spectra have been obtained in the HPLC-DAD-ESI-MS ${ }^{n}$ analyses and the $\mathrm{MS}^{4}$ spectrum in the ESI-MS ${ }^{n}$ direct infusion analyses, which might explain the existence of fragment ions in the latter, which seems not to belong to the fragmentation of ion at $m / z 631$.

However, in order to simplify the discussion, in this paper we will refer to this loss as RDA without taking into account the type of linkage.

In the $\mathrm{MS}^{4}$ analyses, the dimeric aglycones were fragmented. The most abundant fragment ions originated by the loss of $18 \mathrm{amu}$ (water molecule) and $126 \mathrm{amu}$ (phloroglucinol molecule, as previously indicated). In the $\mathrm{MS}^{4}$ spectra, it was also usual to find the ions of the monomeric anthocyanin aglycones, but they were always in lower relative abundances than the former ions, which might indicate that the linkage between anthocyanins is stronger, and therefore, more difficult to break than those of ring $C$ of the upper unit. Although $\mathrm{MS}^{3}$ spectra also provided this information, it is more clearly obtained from the $\mathrm{MS}^{4}$ spectra, since, at this level, only fragmentation of the dimeric aglycone is possible, thus indicating which sites are easier to cleave. The neutral loss of the anthocyanin in the upper or top position is $1 \mathrm{amu}$ lower than the molecular weight of the anthocyanin (302 amu for Dp, 286 for Cy, 316 for Pt, 300 for Pn and 330 for Mv, as can be seen in Table 1), irrespective of being in the flavene or the flavan form. An anthocyanidin in the flavene form possesses one additional proton compared to the flavylium form. However, similar to what is reported for the T- $\mathrm{A}^{+}$ compounds, ${ }^{19,27,31}$ when the flavanol is linked through its $\mathrm{C}_{4}$ position to an anthocyanin and is further fragmented, it is lost as a neutral fragment 2 amu lower than its original weight. When (epi)catechin (molecular weight, 290) is lost, the loss of $288 \mathrm{amu}$ is observed, and when it is (epi)gallocatechin (molecular weight, 306), the loss is 304 . Thus, when a malvidin in the flavene form (molecular weight, 332) is lost, the loss of $330 \mathrm{amu}$ is observed, which is 1 amu lower than the malvidin in flavylium form and 2 amu lower than its molecular weight. On the other hand, an anthocyanidin in flavan form has two additional protons in relation to the flavylium form, but if it is linked through an A-type linkage, one of these protons is replaced by the additional linkage. For this reason, when a flavene linked through a B-type linkage and a flavan linked through an A-type linkage are lost as neutral fragments, the value of the loss is the same in both the cases.

The fragmentation pattern of dimeric anthocyanins containing one glucose and one $p$-coumaroylglucose was very similar to that found for the dimers with two glucoses. The differences in the fragmentation pattern were due to the presence of the $p$-coumaroylglucose moiety instead of the glucose one (Table 1), which was easily lost in the $\mathrm{MS}^{2}$ analyses, either alone (308 amu) or together with the remaining glucose $(470 \mathrm{amu})$. Since the most abundant ion in their $\mathrm{MS}^{2}$ spectra was the same as that originated in the fragmentation of dimeric anthocyanins containing only glucose residues, the fragment ions originated in the 


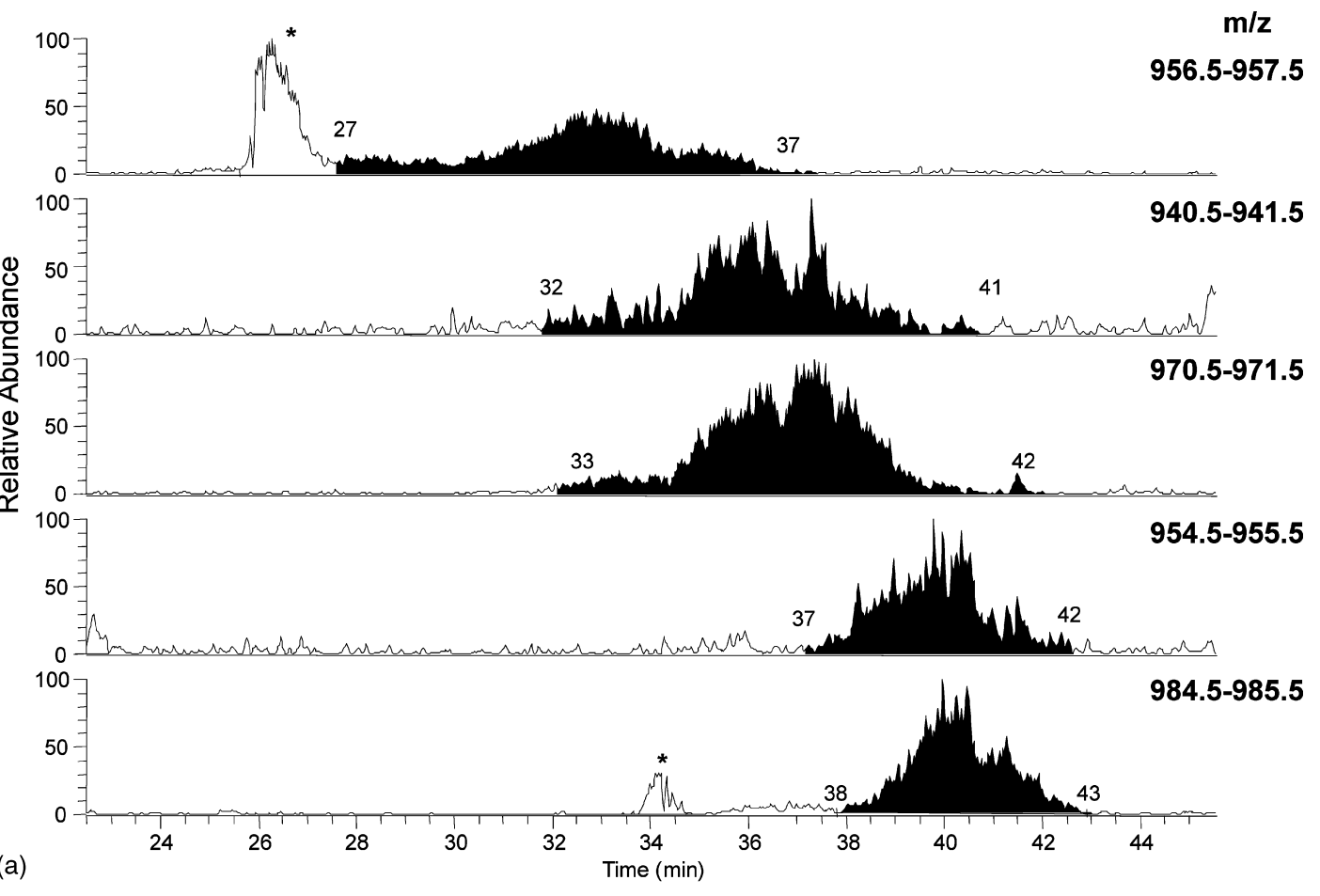

(a)

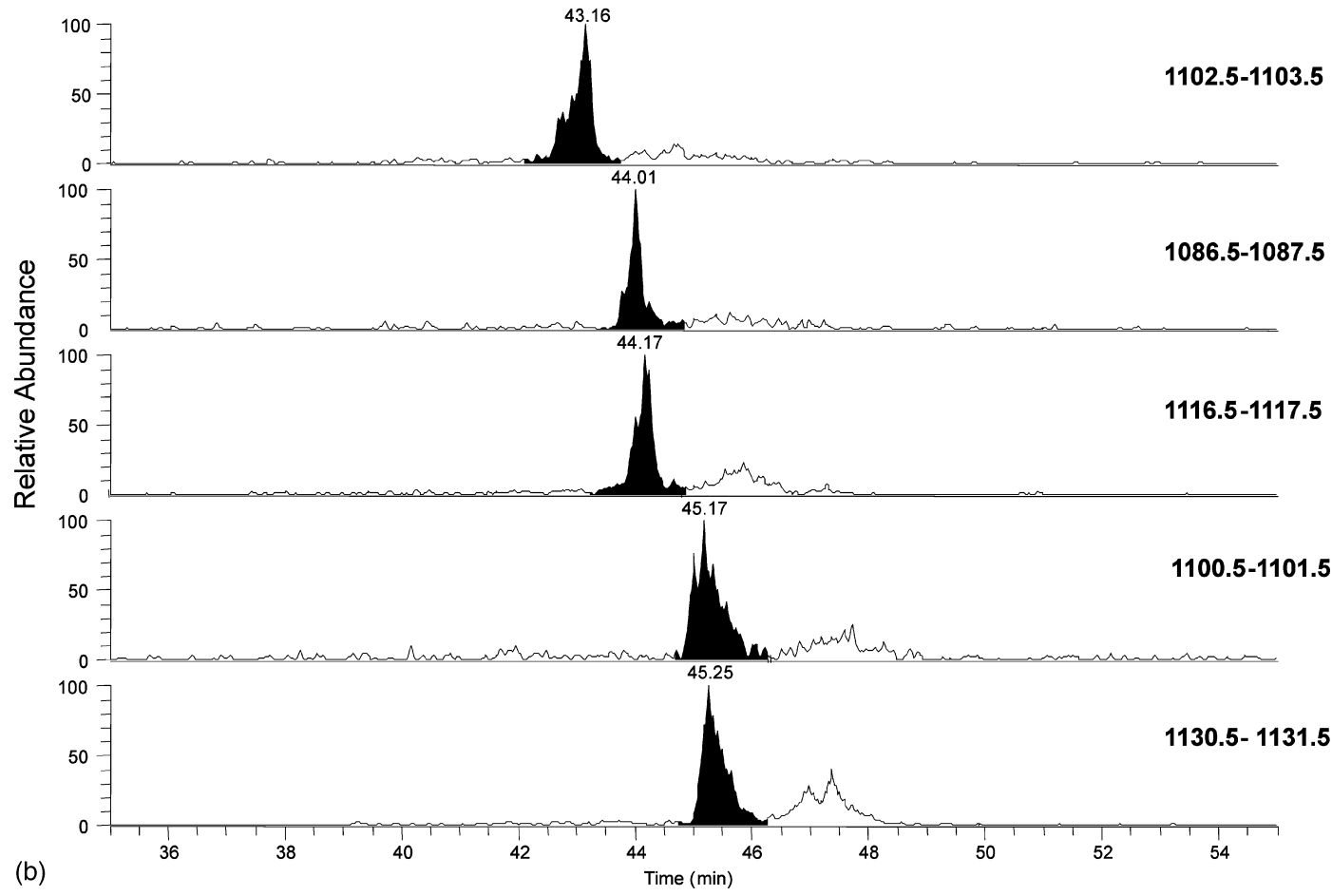

Figure 3. Extracted ion current chromatograms (EIC) of the dimeric anthocyanins detected in this study. (a) Dimeric anthocyanins containing two glucoses. (b) Dimeric anthocyanins containing one glucose and one $p$-coumaroylglucose. The asterisks indicate the signals corresponding to 'non-covalent' dimeric anthocyanins originating in the mass spectrometer during the analysis.

$\mathrm{MS}^{3}$ and $\mathrm{MS}^{4}$ analyses were the same in both types of compounds.

The elution order of all these oligomeric anthocyanins in HPLC was in agreement with the identities proposed for them. Figure 3 shows for these compounds the extracted ion current chromatograms (EIC) from the total ion current (TIC) chromatogram of the fraction mostly composed of anthocyanins of one of the samples analysed. As can be observed, the elution profile of the dimeric anthocyanins containing two glucoses (Fig. 3(a)) seems different from that of the dimers containing one glucose and one $p$-coumaroylglucose (Fig. 3(b)). The former ones eluted as a hump rather than as a peak. In the conditions used, some of these compounds were eluting for $10 \mathrm{~min}$, as was the case of the dimers containing $\mathrm{Mv}$ and Dp, whereas the others took 5-8 min to elute completely from the column. The elution time ranges of these dimers are also shown in Table 1 . The fact that the first dimer 
began elution at approximately $27 \mathrm{~min}$ and that the last one ended at 43 min would partially explain the elevation in the base line observed at the end of the chromatograms of wines when analysed under the same conditions.

In some of the samples where the concentration of the anthocyanin monoglucosides was high, two additional signals appeared in the EIC chromatograms of the ions at $m / z 957$ and 985 (indicated by asterisks in Fig. 3(a)). The first one was observed at the same time of the Pt-3glucoside (26.3 $\mathrm{min}$ ) and the other one at the same time as the Mv-3-glucoside (34.2 min). Their $\mathrm{m} / \mathrm{z}$ ratios corresponded to dimers of the monoglucoside with which they eluted. The formation of non-covalently linked dimers has been reported in other kinds of samples and analytes when employing ESI as the ionisation technique and seems to be related to a high concentration of the analytes. ${ }^{26,36,37}$ Regarding flavonoids, Fulcrand and co-workers ${ }^{36}$ reported the formation of non-covalent dimers of catechin and epicatechin, which eluted at the same time as the monomers, and Vidal and co-workers ${ }^{26}$ also observed this phenomenon in concentrated solutions of anthocyanins when analysed by ESI-MS. Thus, it could be concluded that the signals at the $m / z$ of dimeric anthocyanins, appearing at the same time of their corresponding monomeric anthocyanins would be produced by the 'non-covalent staking' of the monomeric anthocyanins. These complexes might be formed by one anthocyanin in the flavylium form and the other in the quinonoidal form, both forms existing simultaneously at the moment of elution because of the composition of the eluent. Then, the high concentration of each monoglucoside at the moment of the elution would favour the association, and the softness of the ESI technique would allow the detection of these non-covalent complexes. In the conditions employed in our study, it was possible to differentiate which part of the signal corresponded to these 'non-covalent' dimers and which one to the 'covalent' ones, since the elution profiles of the 'non-covalent' ones fitted perfectly with those of the monomeric anthocyanins from which they are formed. Moreover, in the fragmentation of the non-covalent dimer at $m / z$ 957, a fragment ion at $m / z 317$ (Pt) could be observed, instead of those observed in the covalent one at $m / z 331(\mathrm{Mv})$ and 303 (Dp). As far as we could see, the fragmentation of the non-covalent dimers seemed simpler than that of the covalent ones: the loss of the sugars and the loss of one of the subunits were only observed, whereas in the fragmentation of the covalent ones more fragments could be formed. However, more studies have to be done to establish more differences between these two kinds of dimers.

As can be seen in Fig. 3(b), the elution profile of the dimers containing one $p$-coumaroyl moiety appeared as a peak at retention times (calculated at the top of the peak) ranging from minute 43 (dimers containing $\mathrm{Mv}$ and $\mathrm{Dp})$ to minute 45 (dimers containing two $\mathrm{Mv}$ moieties). Nevertheless, as their retention times coincide with those of other compounds that are present in higher amounts, they are not detected as independent peaks in the chromatograms of non-fractionated wines and would, therefore, also contribute to the elevation in the base line of these chromatograms.
In order to verify that all these dimeric pigments were not formed during the fractionation process, the non-fractionated wines were also analysed by HPLC-DADESI/MS ${ }^{n}$. The signals of most of these compounds were observed in the mass spectra, and their EIC chromatograms showed the same elution profile as the corresponding EIC chromatograms obtained in the analyses of the fractions.

\section{Oligomeric pigments: $\mathrm{F}-\mathrm{A}-\mathrm{A}^{+}$}

In the wine fractions that were mostly composed of flavanol-anthocyanin condensation products, a series of ions at $\mathrm{m} / \mathrm{z}$ ranging from 1229 to 1289 were observed. Table 2 reports for each compound the fragment ions that appeared in most of the scans and supplied useful information about the structure of the compound. In addition, other minor fragment ions not shown in Table 2 helped us to confirm the structure. These compounds were present in lower amounts compared to the dimeric anthocyanins and, therefore, hardly any information could be obtained from their $\mathrm{MS}^{4}$, since at this level most signals could not be distinguished from the noise. Only in the case of the compound at $m / z 1259$, the $\mathrm{MS}^{4}$ analyses were useful for compound identification.

The detected compounds were divided into two groups taking into account their elution order and differences in their fragmentation. The most abundant group consisted of compounds showing molecular ions at $m / z 1245,1229,1259$, 1243 and 1273 (cited in elution order). The differences in their $m / z$ were the same as in the five wine anthocyanidins, suggesting that their structures vary in the presence of one anthocyanidin moiety, i.e. Dp, Cy, Pt, Pn or Mv.

As can be seen in Table 2, some of the losses and fragment ions produced were identical to those observed in the fragmentation of the dimeric anthocyanins (Table 1). The most abundant compound showed a signal at $\mathrm{m} / \mathrm{z}$ 1273 , followed by the one with a signal at $m / z 1259$. Their fragmentation supplied more information than those of the other members of the group. The molecular ion at $m / z 1273$ yielded, in the $\mathrm{MS}^{2}$ analysis, five fragment ions (Table 2 and Fig. 4). The most abundant ones derived from the loss of one (ion at $m / z 1111$ ) and two (ion at $m / z$ 949) glucose moieties. The third ion in abundance originated from the loss of $612 \mathrm{amu}$ and possessed the same $\mathrm{m} / \mathrm{z}$ ratio as the $\mathrm{Mv}-\mathrm{Mv}$ aglycone dimer. This loss is explained by the release of the two glucose moieties and a fragment of 288 amu attributed to the loss of a (epi)catechin residue linked to the structure through its $\mathrm{C}_{4}$ position, similar to what is described for the flavanol-anthocyanin direct condensation products. ${ }^{27,31}$ Other fragments observed in the $\mathrm{MS}^{2}$ analysis of this compound $(m / z$ 1273) (Table 2 and Fig. 4) corresponded to the loss of the two glucose moieties and a water molecule ( $-342 \mathrm{amu}, \mathrm{m} / \mathrm{z}$ at 931 ) and to the loss of the (epi)catechin moiety and one glucose $(-450 \mathrm{amu}, \mathrm{m} / \mathrm{z}$ at 823). This latter fragment ion was identical to the major ion originating in the $\mathrm{MS}^{2}$ fragmentation of the Mv-Mv dimers. From this data, it could be proposed that the compound is a trimer containing one (epi)catechin moiety as top unit linked at $\mathrm{C}_{4}$ to a dimeric anthocyanin, composed of two $\mathrm{Mv}$-3-glucosides through the nucleophilic positions $\left(\mathrm{C}_{6}\right.$ or $\mathrm{C}_{8}$ ) of the upper unit of the dimer. As previously discussed 
Table 2. Retention times, molecular and fragment ions of the F-A-A trimers detected in the samples analysed. The fragment ions are listed in order of abundance

\begin{tabular}{|c|c|c|c|c|c|}
\hline$\left(\mathrm{M}^{+}\right) m / z$ & $\begin{array}{c}t_{\mathrm{R}} \\
(\mathrm{min})\end{array}$ & $\begin{array}{c}\mathrm{MS}^{2} \\
\text { fragment ions } \\
m / z\end{array}$ & $\begin{array}{c}\mathrm{MS}^{3} \\
\text { fragment ions } \\
m / z\end{array}$ & $\begin{array}{c}\mathrm{MS}^{4} \\
\text { fragment ions } \\
m / z\end{array}$ & $\begin{array}{l}\text { Proposed } \\
\text { identity }\end{array}$ \\
\hline 1245 & $7-9$ & $\begin{array}{c}1083\left[\mathrm{M}^{+}-162\right] \\
795\left[\mathrm{M}^{+}-450\right] \\
921\left[\mathrm{M}^{+}-324\right] \\
903\left[\mathrm{M}^{+}-342\right]\end{array}$ & $\begin{array}{l}921\left[\mathrm{MS}^{+}-162\right] \\
903\left[\mathrm{MS}^{+}-180\right] \\
657\left[\mathrm{MS}^{+}-426\right] \\
633\left[\mathrm{MS}^{+}-450\right] \\
837\left[\mathrm{MS}^{+}-246\right]\end{array}$ & & (E)C-DpG-MvG \\
\hline 1229 & $9-15$ & $\begin{array}{c}1067\left[\mathrm{M}^{+}-162\right] \\
904\left[\mathrm{M}^{+}-324\right]\end{array}$ & $\begin{array}{l}905\left[\mathrm{MS}^{+}-162\right] \\
917\left[\mathrm{MS}^{+}-150\right]\end{array}$ & & (E)C-CyG-MvG \\
\hline 1259 & $9-16$ & $\begin{array}{c}1097\left[\mathrm{M}^{+}-162\right] \\
971\left[\mathrm{M}^{+}-288\right] \\
935\left[\mathrm{M}^{+}-324\right] \\
809\left[\mathrm{M}^{+}-450\right] \\
1079\left[\mathrm{M}^{+}-180\right]\end{array}$ & $\begin{array}{l}935\left[\mathrm{MS}^{+}-162\right] \\
917\left[\mathrm{MS}^{+}-180\right] \\
899\left[\mathrm{MS}^{+}-198\right] \\
671\left[\mathrm{MS}^{+}-426\right] \\
747\left[\mathrm{MS}^{+}-350\right] \\
971\left[\mathrm{MS}^{+}-126\right]\end{array}$ & $629\left[\mathrm{MS3}^{+}-306\right]$ & (E)C-PtG-MvG \\
\hline 1243 & $12-18$ & $\begin{array}{l}1081\left[\mathrm{M}^{+}-162\right] \\
1063\left[\mathrm{M}^{+}-180\right]\end{array}$ & $919\left[\mathrm{MS}^{+}-162\right]$ & & (E)C-PnG-MvG \\
\hline 1273 & $13-19$ & $\begin{array}{c}1111\left[\mathrm{M}^{+}-162\right] \\
949\left[\mathrm{M}^{+}-324\right] \\
661\left[\mathrm{M}^{+}-612\right] \\
931\left[\mathrm{M}^{+}-342\right] \\
823\left[\mathrm{M}^{+}-450\right]\end{array}$ & $\begin{array}{l}949\left[\mathrm{MS}^{+}-162\right] \\
931\left[\mathrm{MS}^{+}-180\right] \\
823\left[\mathrm{MS}^{+}-288\right] \\
685\left[\mathrm{MS}^{+}-426\right] \\
661\left[\mathrm{MS}^{+}-450\right] \\
737\left[\mathrm{MS}^{+}-374\right] \\
913\left[\mathrm{MS}^{+}-198\right] \\
535\left[\mathrm{MS}^{+}-576\right] \\
331\left[\mathrm{MS}^{+}-780\right]\end{array}$ & & (E)C-MvG-MvG \\
\hline 1261 & $5-7$ & $1099\left[\mathrm{M}^{+}-162\right]$ & $937\left[\mathrm{MS}^{+}-162\right]$ & & (E)GC-DpG-MvG \\
\hline 1275 & $7-9$ & $1113\left[\mathrm{M}^{+}-162\right]$ & $\begin{array}{l}951\left[\mathrm{MS}^{+}-162\right] \\
647\left[\mathrm{MS}^{+}-466\right]\end{array}$ & & (E)GC-PtG-MvG \\
\hline 1289 & $9-14$ & $\begin{array}{c}1127\left[\mathrm{M}^{+}-162\right] \\
965\left[\mathrm{M}^{+}-324\right] \\
661\left[\mathrm{M}^{+}-628\right] \\
823\left[\mathrm{M}^{+}-466\right]\end{array}$ & $\begin{array}{l}965\left[\mathrm{MS}^{+}-162\right] \\
929\left[\mathrm{MS}^{+}-198\right] \\
947\left[\mathrm{MS}^{+}-180\right] \\
823\left[\mathrm{MS}^{+}-304\right] \\
865\left[\mathrm{MS}^{+}-262\right] \\
661\left[\mathrm{MS}^{+}-466\right] \\
535\left[\mathrm{MS}^{+}-592\right] \\
839\left[\mathrm{MS}^{+}-288\right] \\
467\left[\mathrm{MS}^{+}-660\right] \\
331\left[\mathrm{MS}^{+}-796\right]\end{array}$ & & (E)GC-MvG-MvG \\
\hline
\end{tabular}

$\mathrm{M}^{+}$, molecular ion; $\mathrm{t}_{\mathrm{R}}$, retention time for the compounds eluting as peaks, and range of time for the peaks eluting as a hump; MS2 ${ }^{+}$, major fragment ion obtained in the $\mathrm{MS}^{2}$ analysis; $\mathrm{MS3}^{+}$, major fragment ion obtained in the $\mathrm{MS}^{3}$ analysis; Dp, delphinidin; $\mathrm{Cy}$, cyanidin; Pt, petunidin; Pn, peonidin; Mv, malvidin; G, glucose; (E)C, (epi)catechin; (E)GC, (epi)gallocatechin.

for the dimeric anthocyanins, the middle unit could be in the flavene or flavan form, whereas the anthocyanin base unit would be in its flavylium form (Fig. 5(a) and (b)). This type of $\mathrm{F}-\mathrm{A}-\mathrm{A}^{+}$structure might be formed by the reaction of a flavan-3-ol $\mathrm{C}_{4}$ carbocation released after acid-catalysed cleavage of a procyanidin with a dimeric anthocyanin, as described for $\mathrm{F}-\mathrm{A}^{+}$direct condensation products. ${ }^{35}$ To our knowledge, this is the first time that compounds derived from dimeric anthocyanins are described.

The $\mathrm{MS}^{3}$ losses observed for the major $\mathrm{MS}^{2}$ ion $(\mathrm{m} / \mathrm{z}$ 1111 ) allowed the confirmation of the proposed structure.
The major $\mathrm{MS}^{3}$ ions originated from loss of the remaining glucose moiety $(-162 \mathrm{amu}, m / z$ 949) and further loss of a water molecule ( $-18 \mathrm{amu}, \mathrm{m} / \mathrm{z}$ 931). The ion at $\mathrm{m} / \mathrm{z}$ 913 (-198 amu) probably originated from the simultaneous loss of one glucose moiety and two water molecules $(-162-18-18 \mathrm{amu})$. The fragment ions corresponding to the dimeric anthocyanin aglycone $(m / z 661)$ and to the monoglucosilated anthocyanin dimer $(\mathrm{m} / \mathrm{z}$ 823) were also observed in this spectrum, as well as that of the malvidin aglycone $(m / z 331)$. The fragment ions at $m / z 685,737$ and 535 were also helpful in confirming the proposed structure. 

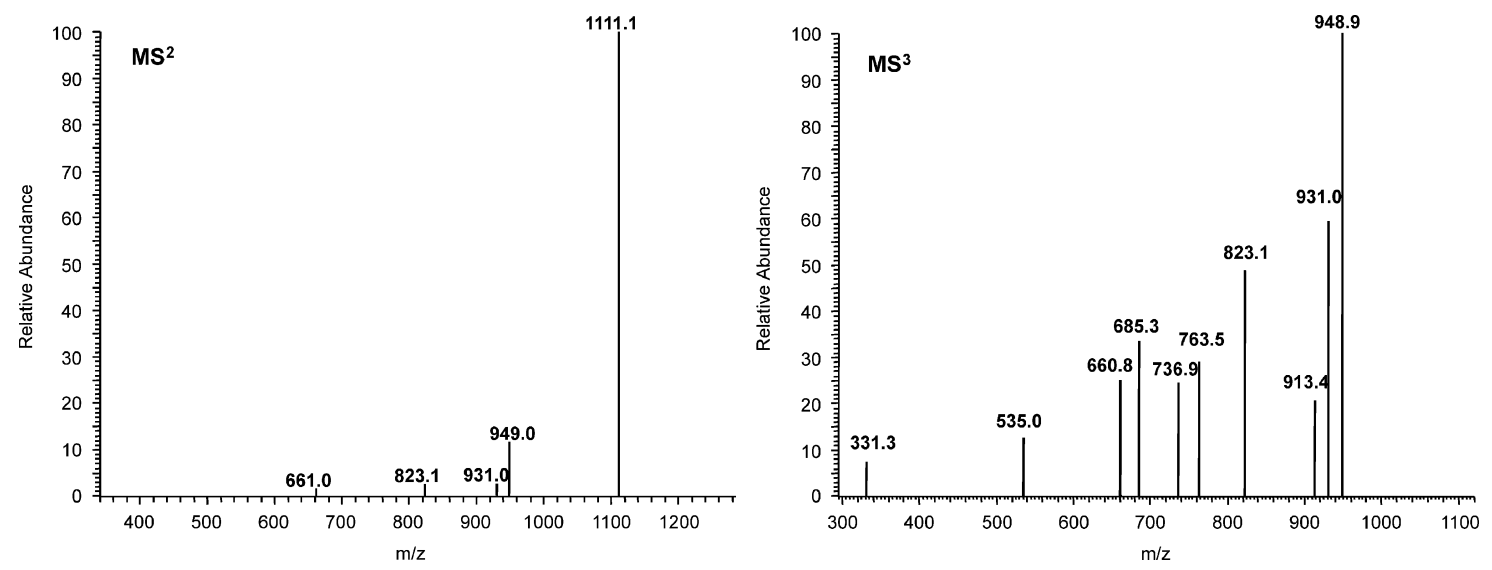

Figure 4. $\mathrm{MS}^{2}$ and $\mathrm{MS}^{3}$ spectra of the trimer (epi)catechin-malvidin-3-glucoside-malvidin-3-glucoside $(\mathrm{m} / \mathrm{z}$ 1273).
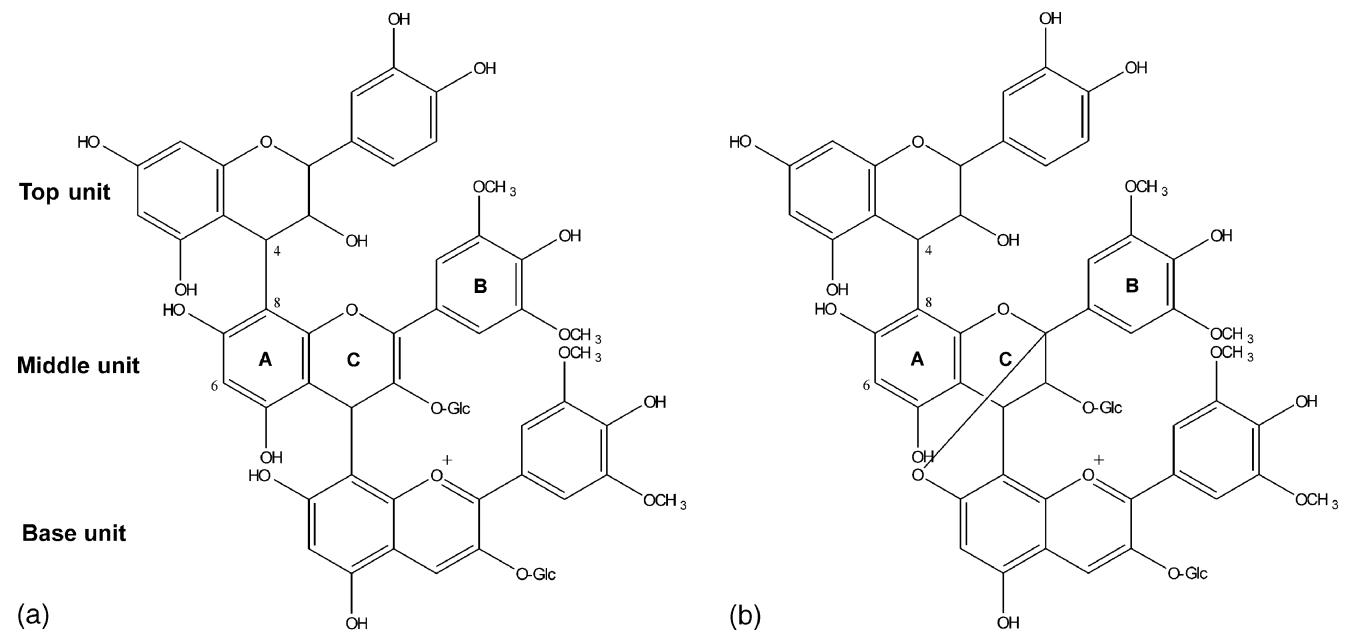

Figure 5. Possible structures for the molecular ion at $m / z$ 1273: (a) (E)C-MvG-Mv ${ }^{+}$G trimer in flavene-flavylium form (B-type linkage). (b) (E)C-MvG-Mv ${ }^{+} \mathrm{G}$ trimer in flavan-flavylium form (A-type linkage).

The first one is produced by the loss of $426 \mathrm{amu}$, which could be due to the losses of the glucose moiety, a molecule of water and a fragment of $246 \mathrm{amu}$. This latter is characteristic of the fragmentation of the $\mathrm{F}-\mathrm{A}^{+}$condensation products containing (epi)catechin ${ }^{27,31}$ and is produced by the cleavage of bonds 2 and 4 of the heterocyclic $C$ ring of the (epi)catechin unit (Fig. 6), thus confirming, along with the loss of $288 \mathrm{amu}$ in the $\mathrm{MS}^{3}$ spectrum $(\mathrm{m} / \mathrm{z}$ 823), the presence of a (epi)catechin unit linked through its $\mathrm{C}_{4}$ position. The fragment ion at $m / z 737(-374 \mathrm{amu})$ is attributed to the RDA fission of $\mathrm{Mv}$ $(-194 \mathrm{amu})$ together with the loss of the glucose moiety and a water molecule. The ion at $m / z 535$ (-576 amu) was also observed in the fragmentation of the dimeric anthocyanin containing two malvidins and two glucoses (Table 1). In the case of the dimeric anthocyanin, it originated in the $\mathrm{MS}^{3}$ analysis owing to the loss of a glucose moiety and the phloroglucinol released after HRF in the upper unit $(-126-162 \mathrm{amu})$. Similarly, the loss of $576 \mathrm{amu}$ can be attributed to the losses of a glucose moiety, the upper (epi)catechin unit and the fragment produced in the HRF of the middle anthocyanidin unit $(-162-288-126 \mathrm{amu})$ (Fig. 6).

In the $\mathrm{MS}^{2}$ fragmentation of the compound showing $\mathrm{m} / \mathrm{z}$ at 1259 (Table 2), the losses observed were similar to those produced in the fragmentation of the previously discussed trimer. The loss of $288 \mathrm{amu}$ produced a fragment ion with the same $m / z$ ratio as the molecular ion of the dimeric anthocyanin containing Pt-3-glc and Mv-3-glc $(\mathrm{m} / \mathrm{z}$ 971), and the major fragment ion, which originated from this dimeric anthocyanin $(m / z$ 809), was also observed. Thus, this compound could be assigned to a trimer containing (epi)catechin linked to the Pt-3-glc-Mv-3-glc dimer. In its $\mathrm{MS}^{3}$ spectra, besides the ions originating after the loss of the remaining glucose, alone or with a water molecule $(m / z$ at 935 and 917, respectively), additional losses were produced in relation to the $\mathrm{MS}^{3}$ fragmentation of the previous trimer. The third ion in abundance was observed at $m / z 899$ (-198 amu), which may also be, as discussed above, due to the loss of the glucose moiety and two water molecules. However, in this case, a second pathway is also possible: the simultaneous loss of a water molecule and the fragment released in the RDA fission of $\mathrm{Pt}(-18-180 \mathrm{amu})$. This would mean that the Pt moiety would be in the flavene or flavan form, otherwise its RDA would not be produced, and that, therefore, it constitutes the middle unit of the trimer. The existence of two possible ways of formation of the ion at $m / z 899$ would explain its higher relative abundance compared to the corresponding fragment 


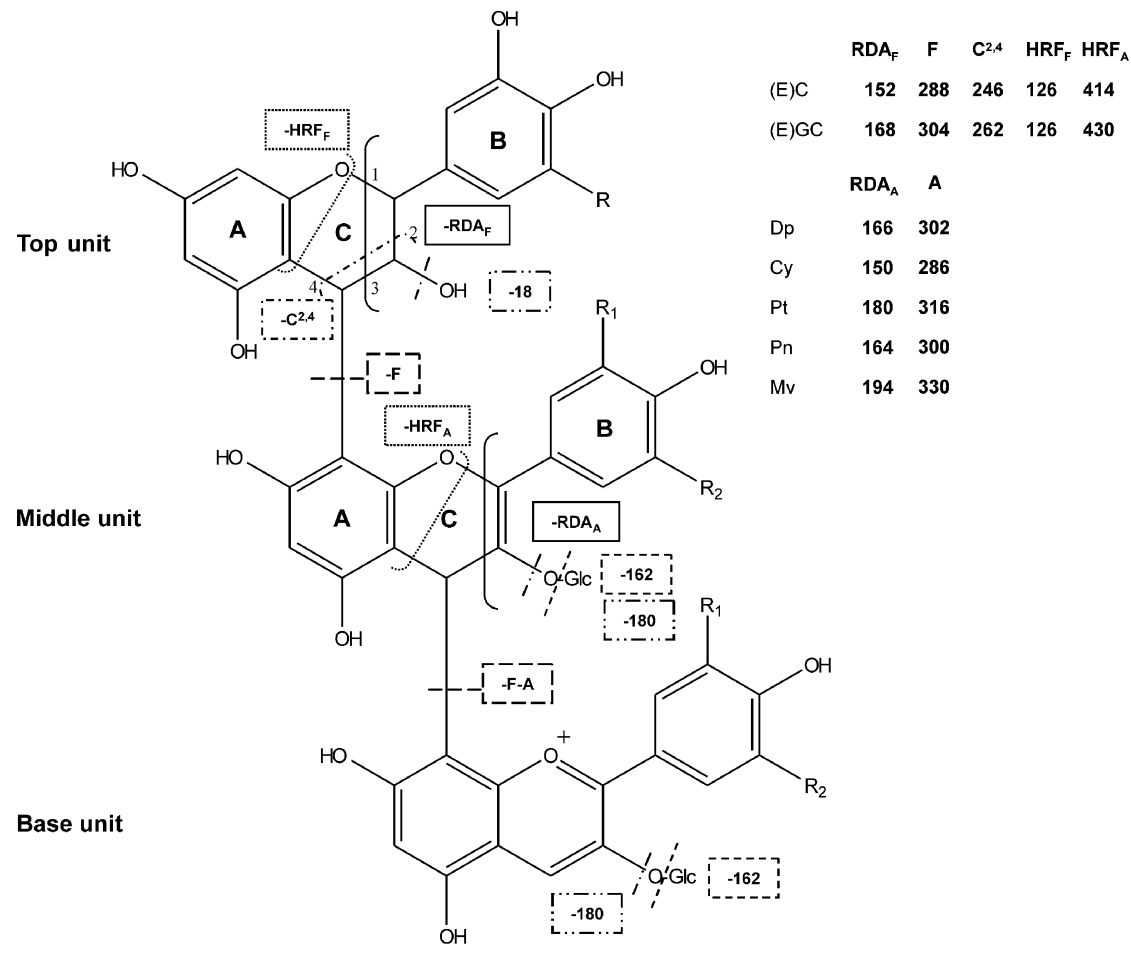

Figure 6. Main fragmentation sites in the F-A-A trimers and the numerical values of the losses observed in the fragmentation. The lines indicating the fragmentation sites and those surrounding the corresponding losses have the same format. (E)C, (epi)catechin; (E)GC, (epi)gallocatechin; Dp, delphinidin; Cy, cyanidin; Pt, petunidin; Pn, peonidin; Mv, malvidin; $\mathrm{R}_{1}$ and $\mathrm{R}_{2}=\mathrm{H}, \mathrm{OH}_{\text {or }} \mathrm{OCH}$, depending on the anthocyanins involved in the compound; $\mathrm{RDA}_{F}$, loss originating from the retro Diels-Alder fission in the flavanol moiety; $\mathrm{RDA}_{\mathrm{A}}$, loss originating from the retro Diels-Alder fission in the anthocyanin moiety; $\mathrm{F}$, flavanol; $\mathrm{A}$, loss observed when the middle anthocyanin unit is lost; $C_{,}^{2,4}$ loss originating after the cleavage of the linkages 2 and 4 of the flavanol; HRF $F_{F}$, loss originating from the heterocyclic ring fission in the flavanol top unit; $\mathrm{HRF}_{\mathrm{A}}$, loss originating from the heterocyclic ring fission in the middle anthocyanin unit.

ion produced from the previously discussed trimer. No observation was made of the fragment corresponding to the RDA fission of $\mathrm{Mv}$, indicating that this trimer originated from the Pt-3-glc(flavene/flavan)-Mv-3-glc(flavylium) dimer and not from Mv-3-glc(flavene/flavan)-Pt-3-glc(flavylium). The presence of a (epi)catechin was confirmed by the loss of $426 \mathrm{amu}$, as already explained for the trimer at $\mathrm{m} / \mathrm{z} 1273$ and by the formation of other two ions at $m / z 747$ and 971, respectively. The first one originated from the loss of 350 amu, attributed to the simultaneous loss of 152 amu and 198 amu corresponding to the RDA fissions in the (epi)catechin and in the Pt units, respectively, accompanied by the loss of water. The second ion, at $m / z 971$, was attributed to the HRF in the (epi)catechin unit (-126 amu) (Fig. 6). In the MS spectrum, only one ion supplying useful information was observed at $m / z 629$ ( $-306 \mathrm{amu}$ ), which could correspond to the RDA fission in the Pt unit together with the (epi)catechin $\operatorname{HRF}(-180-126 \mathrm{amu})$.

The $\mathrm{MS}^{2}$ and $\mathrm{MS}^{3}$ fragmentations of the molecular ions at $m / z 1245,1229$ and 1243 can be explained by the patterns reported above. All these compounds were identified as F$\mathrm{A}-\mathrm{A}^{+}$trimers and considering the conclusions drawn from the fragmentation pattern of the compound at $m / z 1259$, it can be speculated that they consist of an (epi)catechin top unit and a Mv-3-glc base unit differing from each another in the middle anthocyanin unit: Dp-3-glc, Cy-3glc and Pn-3-glc, respectively. The observation of the RDA of Cy $(-150 \mathrm{amu})$ in the trimer at $m / z 1229$ supports this assumption.

A second group of compounds was constituted by the products showing molecular ions at $m / z 1261,1275$ and 1289. Among them, the most abundant was the last one, from which more information regarding fragmentation could be obtained. This compound $(\mathrm{m} / \mathrm{z} 1289)$ showed similar fragmentation pattern (Table 2 and Fig. 7) as the trimer containing an (epi)catechin unit linked to a Mv-3glc dimer $(m / z$ 1273), previously described, from which it differs in 16 amu. Major ions observed in the $\mathrm{MS}^{2}$ and $\mathrm{MS}^{3}$ spectra corresponded to the successive losses of two glucose residues. Similarly, the ions at $m / z 661$ and 823, corresponding to the aglycone and monoglucosilated Mv3-glc dimer, respectively, were observed, confirming that this dimeric anthocyanin is present in the structure of the compound. Thus, the difference of 16 amu could be attributed to the presence of an additional hydroxyl group in the upper or top flavanol unit, and the compound is proposed to be an F$\mathrm{A}-\mathrm{A}^{+}$trimer consisting of (epi)gallocatechin $\mathrm{C}_{4}$-linked to an Mv-3-glc dimer. The losses of 466 amu and $628 \mathrm{amu}$, observed in the $\mathrm{MS}^{2}$ analysis, corresponded to the simultaneous loss of the (epi)gallocatechin residue $(-304 \mathrm{amu})$ and one or two glucose moieties, respectively. The presence of the (epi)gallocatechin was confirmed in the $\mathrm{MS}^{3}$ fragmentation of the major ion in the $\mathrm{MS}^{2}$ spectrum $(m / z 1127)$. The observed loss of $304 \mathrm{amu}$ was two units lower than the molecular 

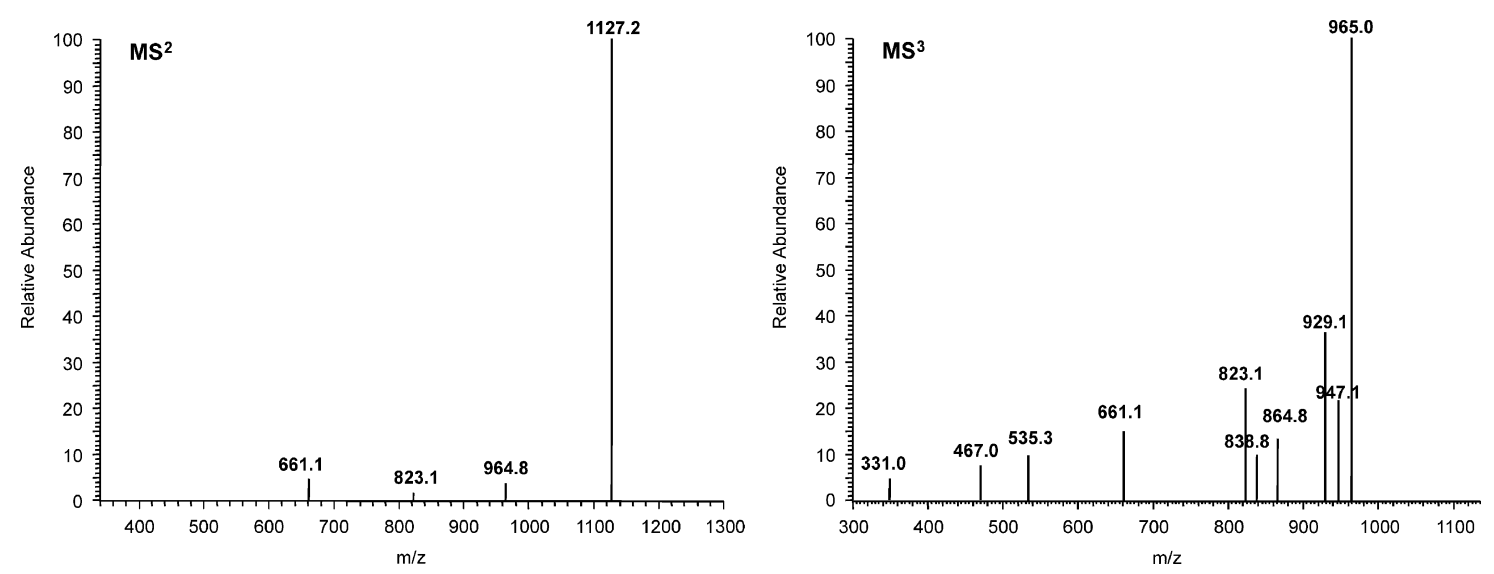

Figure 7. $\mathrm{MS}^{2}$ and $\mathrm{MS}^{3}$ spectra of the trimer (epi)gallocatechin-malvidin-3-glucoside-malvidin-3-glucoside $(\mathrm{m} / \mathrm{z} 1289)$.

mass of (epi)gallocatechin, indicating that it was linked to the rest of the molecule through its $\mathrm{C}_{4}$ position. ${ }^{19}$ The loss of $262 \mathrm{amu}$ corresponded to the cleavage of the bonds 2 and 4 in the $C$ ring of the (epi)gallocatechin, similar to the loss of 246 amu observed for the trimers containing (epi)catechin. The fragment ion at $m / z 467$ (Table 2 and Fig. 7) was also produced in the fragmentation of some dimeric anthocyanins (Table 1) and corresponded to the malvidin linked to the remaining structure of the upper anthocyanin after suffering the RDA fission and losing the glucose moieties. Similarly, in the trimer, it originated from the RDA in the middle anthocyanin (-194 amu, RDA of $\mathrm{Mv})$, accompanied by the losses of the glucose moiety and the (epi)gallocatechin residue $(-194-162-304 \mathrm{amu})$, thus confirming again the presence of two Mv-3-glc and one (epi)gallocatechin units. In this case, the loss of $288 \mathrm{amu}$ producing the fragment ion at $m / z 839$ was not explained by the loss of a (epi)catechin, but to the simultaneous loss of glucose and phloroglucinol released from the HRF in the (epi)gallocatechin unit. The fragment ion at $\mathrm{m} / z 929$ $(-198 \mathrm{amu})$ would correspond to the loss of one glucose moiety and two water molecules. Finally, the fragment ion corresponding to the malvidin aglycone $(\mathrm{m} / \mathrm{z} 331)$ was also detected.

Since the other compounds of this group were present in smaller amounts, only the losses of the sugars were clearly observed in their fragmentation, but their elution order and molecular ions allowed us to propose that they were trimers containing one (epi)gallocatechin moiety linked to the dimeric anthocyanins consisting of Dp-3-glc and Mv-3glc $(m / z$ 1261) or Pt-3-glc and Mv-3-glc $(m / z$ 1275). The loss of $466 \mathrm{amu}$ observed in the $\mathrm{MS}^{3}$ of this latter is explained by the loss of the (epi)gallocatechin residue and a glucose moiety, supporting its proposed identity.

The discovery of this new family of compounds (F-A-A $\mathrm{A}^{+}$) has been possible by the use of the HPLC coupled to mass spectrometry and, above all, by the fragmentations observed in the $\mathrm{MS}^{n}$ analyses. Figure 6 shows the main cleavages that take place in these trimers and the losses originated from them. The fact that there are many possible cleavage sites in these compounds and that different fragments can be lost simultaneously would explain the variety of minor fragment ions that could be observed in consecutive $\mathrm{MS}^{2}$ or $\mathrm{MS}^{3}$ scans of the same $\mathrm{F}-\mathrm{A}-\mathrm{A}^{+}$structure. As occurred in the elution of the dimeric anthocyanins, the EIC chromatograms of these trimers showed a hump rather than definite peaks and unlike the dimeric anthocyanins, the F-A-A ${ }^{+}$trimers eluted in the first part of the chromatogram: just like the $\mathrm{F}-\mathrm{A}^{+}$ direct condensation products. These trimers can be partially responsible for the elevation in the base line observed in the chromatogram of the fractions containing $\mathrm{F}-\mathrm{A}^{+}$direct condensation products.

The presence of these trimers in the non-fractionated wines was also verified, indicating that they are not formed as a consequence of the fractionation process undergone by the wine samples. Nevertheless, as they are in lower concentrations compared to other compounds coeluting with them, their fragmentation pattern could not be obtained in the HPLC-DAD-ESI/MS ${ }^{n}$ analyses of the complete wines. Their elution time ranges obtained through the EIC chromatograms were coincident with those observed for these compounds in the fractions, thus confirming their existence in non-fractionated wines.

\section{CONCLUSIONS}

In this study, the presence of oligomeric anthocyanins in wines has been demonstrated. Moreover, derivatives of these oligomeric anthocyanins have also been detected. ESI/MS and ESI/MS ${ }^{n}$ analyses, coupled or not to HPLC, have supplied the clues to elucidate their structures, which have been interpreted on the basis of the fragmentation pattern established here for the dimeric anthocyanins and considering the previous knowledge on oligomeric anthocyanins and on $\mathrm{F}^{-} \mathrm{A}^{+}$direct condensation products. The newly detected compounds were identified as trimers containing (epi)catechin or (epi)gallocatechin units linked to dimeric anthocyanins. Although it has been possible to establish the identity of the composing units as well as their sequence in the trimers $\left(\mathrm{F}-\mathrm{A}-\mathrm{A}^{+}\right)$, the type of linkage between the anthocyanins is still to be determined. To our knowledge, this is the first time that the existence of these trimers has been reported.

The presence of dimeric anthocyanins and $\mathrm{F}-\mathrm{A}-\mathrm{A}^{+}$ trimers in wine is important because they contribute to the elevation of the base line observed in the chromatograms 
of wines. In the fractionation method used in this work, the dimeric anthocyanins eluted in the fractions mainly composed of monomeric anthocyanins, whereas the F-A-A ${ }^{+}$ trimers eluted with the $\mathrm{F}-\mathrm{A}^{+}$direct condensation products. Since the dimeric anthocyanins were first detected in grape skin, they could be extracted into the must in a similar way as anthocyanins. However, $\mathrm{F}-\mathrm{A}-\mathrm{A}^{+}$trimers might have originated in the wine by a mechanism similar to that proposed for the formation of $\mathrm{F}-\mathrm{A}^{+}$direct condensation products. It might also be expected to find in wine, oligomers of this kind, in which the dimeric anthocyanins would react with oligomeric proanthocyanidins or maybe other kinds of dimeric anthocyanins derivatives, in which the oligomeric anthocyanins would act as substrates of the usual reactions undergone by monomeric anthocyanins.

\section{Acknowledgements}

This work was supported by grant AGL-2005-07245-C03-01 from MEC (Ministerio de Educación y Ciencia, Spain). The authors thank the MECD (Ministerio de Educación, Cultura y Deporte, Spain) for an FPU predoctoral scholarship to C. Alcalde-Eon, and Bodegas RODA (Haro, La Rioja, Spain) for supplying the wine samples. We also thank Dr Pérez-Alonso from our research group for his helpful comments and analytical advice and Dr Boido from the enology section of Universidad de la República in Montevideo (Uruguay) for technical assistance.

\section{REFERENCES}

1. Somers TC. The polymeric nature of wine pigments. Phytochemistry 1971; 10: 2175, DOI: 10.1016/s0031-9422(00)972157.

2. Ribéreau-Gayon P. In Anthocyanins as Food Colors, Markakis P (ed.). Academic Press: New York, 1982; 209.

3. Wulf LW, Nagel CW. High-pressure liquid chromatographic separation of anthocyanins of Vitis vinifera. Am. J. Enol. Vitic. 1978; 29: 42.

4. Williams M, Hrazdina G, Wilkinson MM, Sweeny JG, Iacobucci GA. High-pressure liquid chromatographic separation of 3-glucosides, 3, 5-diglucosides, 3-(6-O-p-coumaryl)glucosides and 3-(6-O-p-coumarylglucoside)-5-glucosides of anthocyanidins. J. Chromatogr. 1978; 155: 389, DOI: 10.1016/s00219673(00)88000-0.

5. Hebrero E, Santos-Buelga C, Rivas-Gonzalo JC. High performance liquid chromatography-diode array spectroscopy identification of anthocyanins of Vitis vinifera variety Tempranillo. Am. J. Enol. Vitic. 1988; 39: 227.

6. Mazza G, Fukumoto L, Delaquis P, Girard B, Ewert B. Anthocyanins, phenolics, and color of cabernet franc, merlot, and pinot noir wines from British Columbia. J. Agric. Food Chem. 1999; 47: 4009, DOI: 10.1021/jf990449f.

7. Revilla E, García-Beneytez E, Cabello F, Martín-Ortega G, Ryan JM. Value of high-performance liquid chromatographic analysis of anthocyanins in the differentiation of red grape cultivars and red wines made from them. J. Chromatogr. A 2001; 915: 53, DOI: 10.1016/s0021-9673(01)00635-5.

8. Strack D, Wray V. In The Flavonoids. Advances in Research Since 1986, Harborne JB (ed.). Chapman and Hall: London, 1994; 1.

9. Baldi A, Romani A, Mulinacci N, Vincieri FF, Casetta B. HPLC/MS application to anthocyanins of Vitis vinifera L. J. Agric. Food Chem. 1995; 43: 2104.

10. Favretto D, Flamini R. Application of electrospray ionization mass spectrometry to the study of grape anthocyanins. Am. J. Enol. Vitic. 2000; 51: 55.

11. Flamini R. Some advances in the knowledge of grape, wine and distillates chemistry as achieved by mass spectrometry. J. Mass Spectrom. 2005; 40: 705, DOI: 10.1002/jms.887.
12. Oliveira MC, Esperança P, Almoster Ferreira MA. Characterisation of anthocyanidins by electrospray ionisation and collisioninduced dissociation tandem mass spectrometry. Rapid Commun. Mass Spectrom. 2001; 15: 1525, DOI: 10.1002/rcm.400.

13. Vivar-Quintana AM, Santos-Buelga C, Rivas-Gonzalo JC. Anthocyanin-derived pigments and colour of red wines. Anal. Chim. Acta 2002; 458: 147, DOI: 10.1016/s0003-2670(01)01619-1.

14. Monagas M, Núñez V, Bartolomé B, Gómez-Cordovés C. Anthocyanin derived pigments in Graciano, Tempranillo and Cabernet Sauvignon wines produced in Spain. Am. J. Enol. Vitic. 2003; 54: 163.

15. Wang H, Race EJ, Shrikhande AJ. Anthocyanin transformation in Cabernet Sauvignon wine during aging. J. Agric. and Food Chem. 2003; 51: 7989, DOI: 10.1021/jf034501q.

16. de Villiers A, Vanhoenacker G, Majek P, Sandra P. Determination of anthocyanins in wine by direct injection liquid chromatography-diode array detection-mass spectrometry and classification of wines using discriminant analysis. J. Chromatogr. A 2004; 1054: 195, DOI: 10.1016/j.chroma.2004.07.087.

17. Alcalde-Eon C, Escribano-Bailón MT, Santos-Buelga C, RivasGonzalo JC. Changes in the detailed pigment composition of red wine during maturity and ageing. A comprehensive study. Anal. Chim. Acta 2006; 563: 238, DOI: 10.1016/j.aca.2005.11.028.

18. Boido E, Alcalde-Eon C, Carrau F, Dellacassa E, RivasGonzalo JC. Aging effect on the pigment composition and color of Vitis vinifera L. cv. Tannat wines. Contribution of the main pigment families to wine color. J. Agric. Food Chem. 2006; 54: 6692, DOI: 10.1021/jf061240m.

19. Alcalde-Eon C, Boido E, Carrau F, Dellacassa E, RivasGonzalo JC. Pigment profiles in monovarietal wines produced in Uruguay. Am. J. Enol. Vitic. 2006; 57: 449.

20. Pati S, Losito I, Gambacorta G, La Notte E, Palmisano F, Zambonin PG. Simultaneous separation and identification of oligomeric procyanidins and anthocyanin-derived pigments in raw red wine by HPLC-UV-ESI-MS ${ }^{n}$. J. Mass Spectrom. 2006; 41: 861, DOI: $10.1002 /$ jms.1044.

21. Francia-Aricha EM, Guerra MT, Rivas-Gonzalo JC, SantosBuelga C. New anthocyanin pigments formed after condensation with flavanols. J. Agric. Food Chem. 1997; 45: 2262, DOI: 10.1021/jf9609587.

22. Mateus N, de Pascual-Teresa S, Rivas-Gonzalo JC, SantosBuelga $C$, de Freitas V. Structural diversity of anthocyaninderived pigments in port wines. Food Chem. 2002; 76: 335, DOI: 10.1016/s0308-8146(01)00281-3.

23. Mateus N, Silva AMS, Rivas-Gonzalo JC, Santos-Buelga C, de Freitas V. A new class of blue anthocyanin-derived pigments isolated from red wines. J. Agric. Food Chem. 2003; 51: 1919, DOI: 10.1021/jf020943a.

24. Es-Safi NE, Fulcrand H, Cheynier V, Moutounet M. Studies on the acetaldehyde-induced condensation of (-)-epicatechin and malvidin 3-O-glucoside in a model solution system. J. Agric. Food Chem. 1999; 47: 2096, DOI: 10.1021/jf9806309.

25. Hayasaka Y, Kennedy JA. Mass spectrometric evidence for the formation of pigmented polymers in red wine. Aust. J. Grape Wine Res. 2003; 9: 210.

26. Vidal S, Meudec E, Cheynier V, Skouroumounis G, Hayasaka Y. Mass spectrometric evidence for the existence of oligomeric anthocyanins in grape skins. J. Agric. Food Chem. 2004; 52: 7144, DOI: 10.1021/jf048939h.

27. Alcalde-Eon C, Escribano-Bailon MT, Santos-Buelga C, RivasGonzalo JC. Separation of pyranoanthocyanins from red wine by column chromatography. Anal. Chim. Acta 2004; 513: 305, DOI: 10.1016/j.aca.2003.10.076.

28. Salas E, Dueñas M, Schwarz M, Winterhalter P, Cheynier V, Fulcrand H. Characterization of pigments from different High Speed Countercurrent Chromatography wine fractions. J. Agric. Food Chem. 2005; 53: 4536, DOI: 10.1021/jf0478096.

29. Remy S, Fulcrand H, Labarbe B, Cheynier V, Moutounet M. First confirmation in red wine of products resulting from direct anthocyanin-tannin reactions. J. Sci. Food Agric. 2000; 
80: 745, DOI: 10.1002/(SICI)1097-0010(20000501)80:6<745::AIDJSFA611>3.0.CO;2-4.

30. Remy-Tanneau S, Le Guernevé C, Meudec E, Cheynier V. Characterization of a colorless anthocyanin-flavan-3-ol dimer containing both carbon-carbon and ether interflavanoid linkages by NMR and mass spectrometry. J. Agric. Food Chem. 2003; 51: 3592, DOI: 10.1021/jf021227b.

31. González-Paramás AM, Lopes da Silva F, Martín-López P, Macz-Pop G, González-Manzano S, Alcalde-Eon C, PérezAlonso JJ, Escribano-Bailón MT, Rivas-Gonzalo JC, SantosBuelga C. Flavanol-anthocyanin condensed pigments in plant extracts. Food Chem. 2006; 94: 428, DOI: 10.1016/j.foodchem.2004.11.037.

32. Friedrich W, Eberhardt A, Galensa R. Investigation of proanthocyanidins by HPLC with electrospray ionization mass spectrometry. Eur. Food Res. Technol. 2000; 211: 56, DOI: $10.1007 / \mathrm{s} 002170050589$.

33. Hayasaka Y, Waters EJ, Cheynier V, Herderich MJ, Vidal S. Characterization of proanthocyanidins in grape seeds using electrospray mass spectrometry. Rapid Commun. Mass Spectrom. 2003; 17: 9, DOI: 10.1002/rcm.869.

34. Gu L, Kelm MA, Hammerstone JF, Zhang Z, Beecher G, Holden J, Haytowitz D, Prior RL. Liquid chromatographic/electrospray ionization mass spectrometric studies of proanthocyanidins in foods. J. Mass Spectrom. 2003; 38: 1272, DOI: $10.1002 /$ jms.541.

35. Salas E, Atanasova V, Poncet-Legrand C, Meudec E, Mazauric JP, Cheynier V. Demonstration of the occurrence of flavanolanthocyanin adducts in wine and in model solutions. Anal. Chim. Acta 2004; 513: 325, DOI: 10.1016/j.aca.2003.11.084.

36. Fulcrand H, Remy S, Souquet JM, Cheynier V, Moutounet M. Study of wine tannin oligomers by on-line liquid chromatography electrospray ionization mass spectrometry. $J$. Agric. Food Chem. 1999; 47: 1023, DOI: 10.1021/jf9805496.

37. Niessen WMA. Liquid Chromatography-Mass Spectrometry, Revised and Expanded, 2nd edn. Marcel Dekker: New York, 1999; 634. 